\title{
CCXLIII.-p-Dithiobenzoic Acid.
}

\section{By Samuel Smiles and Douglas Creese Harrison.}

INVESTIGATION of $o$ - and $m$-dithiobenzoic acids has shown that the reactions of the dithio-group in these substances are remarkably different and it thus became desirable to examine the behaviour of the para-isomeride. Although the ortho-derivative has been the subject of much investigation, $p$-dithiobenzoic acid has not yet been mentioned in literature, in fact, the only derivative of the type described seems to be the $p$-methylthiolbenzoic acid (IV) which was obtained by Zincke and Jörg (Ber., 1910, 43, 3448) from $p$-methylthiolaniline by replacement of the amino-group with carboxyl. In the preparation of $m$-dithiobenzoic acid (T., $1921,119,1792)$, reduction of $m$-chlorosulphonylbenzoic acid proved to be the best method of obtaining the material in large quantity; a similar process has been found to be convenient for obtaining the desired para-compound. Technical $p$-toluenesulphonyl chloride serves as a convenient starting material; attempts were made to convert this substance directly to the desired $p$-chlorosulphonylbenzoic acid by oxidation with chromic anhydride in acetic acid solution, but the yields were poor and variable. The chloride was therefore converted to the sodium sulphonate, and this was oxidised in the usual manner, the resulting $p$-sulphobenzoic acid being isolated as the mono-sodium salt (I). The conversion 
of this product to $p$-chlorosulphonylbenzoic acid (II) was readily accomplished by the action of hot chlorosulphonic acid. This method has obvious advantages over the usual method involving the use of phosphorus pentachloride : the partial hydrolysis of the dichloride is avoided, the crude product is sufficiently pure for immediate further treatment, and the yield is almost theoretical. Reduction of this chlorosulphonyl derivative gave good yields of the sulphinic acid (III) or of the corresponding thiol. Methylation of the latter gave the $p$-methylthiolbenzoic acid described by Zincke and Jörg (loc. cit.) and by Gattermann (Annalen, 1912, 393, 226), whilst oxidation readily furnished p-dithiobenzoic acid. It has been previously recorded (T., 1921, 119, 1795) that the dithiogroup in $m$ - and $o$-dithiobenzoic acids is resolved by the action of alkali hydroxide; preliminary experiments with the para-derivative indicate that a similar action takes place and that the fission is much more rapid than in the case of the ortho-compound, which is undoubtedly the least reactive of the three isomerides.

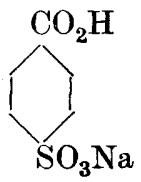

(I.)

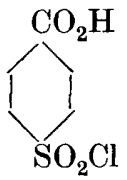

(II.)

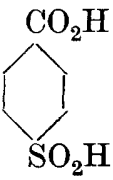

(III.)

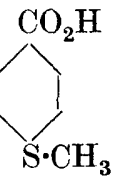

(IV.)

EXPERIM E N T A L.

p-Chlorosulphonylbenzoic Acid, $\mathrm{CO}_{2} \mathrm{H} \cdot \mathrm{C}_{6} \mathrm{H}_{4} \cdot \mathrm{SO}_{2} \mathrm{Cl}$.-The solution obtained by hydrolysis of 51 grams of $p$-toluenesulphonyl chloride with a slight excess of aqueous sodium hydroxide was nearly neutralised with dilute sulphuric acid and then mixed with 88 grams of potassium permanganate. Water was added to bring the volume of the solution to about $1 \frac{1}{4}$ litres, and this was heated until oxidation was complete. The oxides of manganese were removed and the colourless liquid was evaporated to about onethird of the original volume. After being mixed with excess of sulphuric acid, the solution was cooled, when the mono-sodium salt of $p$-sulphobenzoic acid separated. A further quantity of the product was obtained from the liquor by the addition of saturated brine, the total yield being 50 grams of crude material. A sample was purified by crystallisation, first from brine and then from hot water, in which it is freely soluble. Mono-sodium p-sulphobenzoate $\mathrm{CO}_{2} \mathrm{H} \cdot \mathrm{C}_{6} \mathrm{H}_{4} \cdot \mathrm{SO}_{3} \mathrm{Na}, 2 \mathrm{H}_{2} \mathrm{O}$, after being dried in the air, contains 2 mols. of water of crystallisation, which are lost at $125-130^{\circ}$ (Found: $\mathrm{H}_{2} \mathrm{O}=14 \cdot 0 ; \mathrm{Na}$, in anhydrous substance, $=10 \cdot 2$. Calc., 
$\mathrm{H}_{2} \mathrm{O}=13 \cdot 8 ; \mathrm{Na}=10.27$ per cent.). This salt has been previously described as containing two and a half molecules (Annalen, 1875, $178,279)$ and one molecule (Ber., 1877, 10, 1715) of water of crystallisation.

Thirty-five grams of this anhydrous salt were heated with 50 c.c. of chlorosulphonic acid at $100^{\circ}$ for about one hour, then the liquid was cooled and poured slowly over crushed ice. The colourless product was collected, washed with water, and dried; the yield was 30-35 grams. It may here be mentioned that in other experiments which are in progress it has been found that, in contrast with the meta- and para-compounds, o-chlorosulphonylbenzoic acid is very unstable and is readily decomposed by water in the neighbourhood of $0^{\circ}$. Hence it is evident that any of this ortho-compound which might have survived from the technical sulphonyl chloride used is at this stage eliminated. $p$-Chlorosulphonylbenzoic acid is moderately soluble in hot acetone, from which it separates in colourless needles; these melt and decompose at about $235^{\circ}$, the exact temperature depending on the rate of heating (Found: $\mathrm{Cl}=15 \cdot 87$. Calc., $\mathrm{Cl}=16.09$ per cent. For complete interaction, 100 c.c. of $N-\mathrm{NaOH}$ required 7.43 ; calc., $7 \cdot 35$ grams).

p-Sulphinobenzoic Acid, $\mathrm{CO}_{2} \mathrm{H} \cdot \mathrm{C}_{6} \mathrm{H}_{4} \cdot \mathrm{SO}_{2} \mathrm{H}$.-Twenty grams of $p$-chlorosulphonylbenzoic acid were suspended in 250 c.c. of water containing 70 grams of sodium sulphite. The mixture was shaken until all the chloride had dissolved, the liquid being kept weakly alkaline by repeated addition of small quantities of aqueous sodium hydroxide. When interaction was complete, excess of dilute sulphuric acid was added to the mixture, which was then kept for some hours to allow complete separation of the sulphinic acid. This was purified by crystallisation from hot water. p-Sulphinobenzoic acid forms colourless needles which are sparingly soluble in cold water and melt and decompose at about $245^{\circ}$ according to the rate of heating (Found : $\mathrm{S}=17 \cdot 57 . \quad \mathrm{C}_{7} \mathrm{H}_{6} \mathrm{O}_{4} \mathrm{~S}$ requires $\mathrm{S}=17 \cdot 2$ per cent. 100 c.c. of $N \cdot \mathrm{NaOH}$ required 9.4 ; calc., $9 \cdot 3$ grams).

p-Thiolbenzoic Acid, $\mathrm{CO}_{2} \mathrm{H} \cdot \mathrm{C}_{6} \mathrm{H}_{4} \cdot \mathrm{SH}$. - Twenty grams of the carefully dried sulphonyl chloride were dissolved in about 75 c.c. of acetic acid. To the hot solution zinc dust and concentrated hydrochloric acid were added at intervals in small quantities. When reduction was nearly complete, the liquid became turbid, and ultimately a solid separated which increased in quantity after the liquid had been cooled and kept for a few hours. Hydrochloric acid was then added to remove excess of zinc, and the product was collected. About 11 grams of the dried thiol derivative were obtained. The product thus isolated formed a colourless, micro- 
crystalline powder which decomposed at about $250^{\circ}$ and was very sparingly soluble in all common solvents. The latter property combined with the liability of mercaptans to undergo oxidation renders the purification of the substance very difficult. For example, the substance is sparingly soluble in boiling nitrobenzene, but the crystalline material which separates from this solution was found by analysis to be a mixture of the disulphide with other oxidation products. Hence, for analysis, a sample of the substance was submitted to methylation with methyl sulphate in alkaline solution. The product after crystallisation from hot water melted at $190^{\circ}$; Zincke and Jörg give $192^{\circ}$ (loc. cit.), whilst Gattermann (loc. cit.) quotes $190^{\circ}$ as the melting point of $p$-methylthiolbenzoic acid (Found : $\mathrm{C}=56.9 ; \mathrm{H}=5 \cdot 0 ; \mathrm{S}=19.5$. Calc., $\mathrm{C}=57 \cdot 1$; $\mathbf{H}=4.8 ; \mathrm{S}=19.1$ per cent.). The barium, silver, and copper salts of $p$-thiolbenzoic acid are sparingly soluble in water; they exist in two series, in one of which the carboxyl group alone is involved, whilst in the other both this and the thiol group take part. The salts of the latter type are the more soluble.

p-Dithiobenzoic Acid, $\left[\mathrm{CO}_{2} \mathrm{H}^{\circ} \mathrm{C}_{6} \mathrm{H}_{4} \cdot \mathrm{S} \cdot\right]_{2}$. - The dithio-acid is readily formed from the mercaptan by the usual methods of oxidation; it was most readily obtained by heating the latter substance in air at $100^{\circ}$. To prepare a pure sample of the dithio-acid the mercaptan was first purified by the following process. The crude material was dissolved in a slight excess of dilute ammonia, and the solution boiled until nearly neutral; the precipitate, which contained a small quantity of zinc, was removed and the mercaptan liberated by dilute hydrochloric acid. The product was again submitted to the same treatment and then boiled with water until all ammonium chloride was removed. At this stage oxidation to the disulphide was extensive, but the change was completed by heating the substance at $100^{\circ}$ for some hours (Found : $S=21 \cdot 1$; $\mathrm{C}=54 \cdot 7 ; \quad \mathrm{H}=3 \cdot 6 . \quad \mathrm{C}_{14} \mathrm{H}_{10} \mathrm{O}_{4} \mathrm{~S}_{2}$ requires $\mathrm{S}=20.9 ; \quad \mathrm{C}=54.9$; $\mathrm{H}=3 \cdot 2$ per cent.). $\quad p$-Dithiobenzoic acid, prepared in this manner, formed a colourless powder which melted and decomposed somewhat above $320^{\circ}$ and was insoluble in water and very slightly soluble in organic media. The following data illustrate the action of alkali hydroxide with the substance. A sample was dissolved in excess of cold $N / 2$-sodium hydroxide; the free alkali was then estimated after the lapse of ten minutes and it was found that one molecular proportion of the disulphide had combined with $2 \cdot 2$ equivalents of sodium hydroxide. Under these conditions, the dithio-group evidently remains intact. A similar operation with a larger excess of alkali at $100^{\circ}$ showed that decomposition was extensive, since 3.97 equivalents of alkali had combined with one 
2026 MASTERS : REACTIONS OF CELLULOSE WITH SODIUM CHLORIDE molecular proportion of the disulphide. Further experiments are required to show the exact relative stability of the three isomeric dithiobenzoic acids.

Kina's College,

LONDON.

[Received, August 24th, 1922.] 